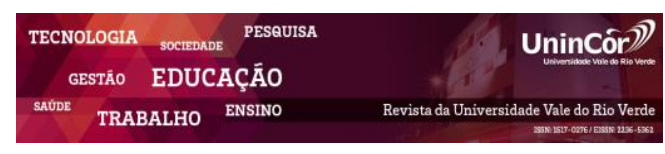

Revista da Universidade Vale do Rio Verde ISSN: 1517-0276 / EISSN: 2236-5362 v. 17 |n. 1 | Ano 2019

Paola Aparecida Alves Ferreira Programa de Mestrado em Saúde, Sociedade e Ambiente da Universidade Federal dos Vales do Jequitinhonha e Mucuri - UFVJM, paola.dtna@gmail.com

Emerson Cotta Bodevan

Universidade Federal dos Vales do Jequitinhonha e Mucuri - UFVJM

ecbodevan@yahoo.com.br

Leida Calegário de Oliveira Universidade Federal dos Vales do Jequitinhonha e Mucuri - UFVJM leida.calegario1@gmail.com

\section{CARACTERÍSTICAS SOCIODEMOGRÁFICAS ASSOCIADAS À PREVALÊNCIA DE HIPERTENSÃO ARTERIAL SISTÊMICA}

\section{RESUMO}

A Hipertensão Arterial Sistêmica é uma Doença Crônica Não Transmissível de grande magnitude e um problema grave de saúde pública mundial $\mathrm{O}$ presente estudo teve como objetivo analisar as características sociodemográficas, relacionando-as com a prevalência de Hipertensão Arterial. Trata-se de um estudo transversal de base populacional (parecer $n^{\circ} 357.240$ ) quantitativo, com aplicação de questionário padronizado do Ministério da Saúde - Vigitel - 2011, com adaptações. Participaram da pesquisa 571 indivíduos na faixa etária de 18 a 46 anos, cadastrados nas Estratégias de Saúde da Família do município Diamantina, MG. Os participantes foram recrutados aleatoriamente, através de sorteio, levando-se em consideração a proporção de moradores por bairro. Para a entrada dos dados, foi utilizado o programa Excel 2010 e para as análises, o aplicativo R Core Team (2015). Foi adotado o nível de significância de $95 \%$. A associação entre a prevalência de hipertensão e as variáveis foi avaliada por meio do Teste de Qui-quadrado de Pearson ou pelo Teste Exato de Fisher com nível de significância de 0,05. A análise dos dados permite-nos observar que a maioria era do sexo feminino $(56,9 \%)$, solteiros $(61,2 \%)$, da raça parda $(57,7 \%)$, na faixa etária entre de 18 a 34 anos (7,6\%), com predomínio do ensino médio completo $(34,8 \%)$. Houve maior prevalência de hipertensão nos indivíduos do sexo feminino (12,0\%), viúvos $(25,0 \%)$ com idade igual ou superior a 45 anos $(51,4 \%)$, menor escolaridade $(39,0 \%)$ e em indivíduos negros $(11,3 \%)$. A prevalência de hipertensão na população estudada foi de $9,6 \%$ (autorreferida).

Palavras-chave: Prevalência. Hipertensão Arterial. Perfil Sociodemográfico. Doença Crônica não Transmissível. Vigitel.

\section{SOCIODEMOGRAPHIC CHARACTERISTICS ASSOCIATED WITH THE PREVALENCE OF SYSTEMIC ARTERIAL HYPERTENSION}

\begin{abstract}
Systemic Arterial Hypertension is a major noncommunicable chronic disease and a serious global public health problem. The present study aimed to analyze the sociodemographic characteristics, relating them to the prevalence of arterial hypertension. This is a population-based cross-sectional study (opinion no. 357,240), with a standardized questionnaire from the Ministry of Health - Vigitel - 2011, with adaptations. A total of 571 individuals in the age range of 18 to 46 years, enrolled in the Family Health Strategies of Diamantina, MG, participated in the study. Participants were recruited randomly, by lot, taking into account
\end{abstract}


the proportion of residents per neighborhood. For the data entry, the Excel 2010 program was used and for the analysis, the R Core Team application (2015). The level of significance was adopted at $95 \%$. The association between the prevalence of hypertension and the variables was assessed using the Pearson's Chi-square test or the Fisher's exact test with a significance level of 0.05 . Data analysis showed that the majority were female $(56.9 \%)$, single $(61.2 \%)$, brown $(57.7 \%)$, in the age group $18-34$ years old $(7.6 \%)$, with a predominance of complete secondary education $(34.8 \%)$. There was a higher prevalence of hypertension in female subjects $(12.0 \%)$, widowers $(25.0 \%)$ with 45 years of age or older $(51.4 \%)$, lower schooling $(39.0 \%)$ and in individuals blacks $(11.3 \%)$. The prevalence of hypertension in the study population was $9.6 \%$ (self-reported).

Keywords: Prevalence. Arterial hypertension. Sociodemographic Profile. Chronic noncommunicable disease. Vigitel.

Recebido em: 12/18/2019 - Aprovado em: 20/03/2019 - Disponibilizado em: 15/07/2019

\section{INTRODUÇÂO}

O aumento da prevalência das Doenças Crônicas Não Transmissíveis (DCNT) é uma das principais características do processo de transição epidemiológica, ocorrido primeiramente nos países desenvolvidos e que vem ocorrendo de maneira rápida no Brasil a partir da década de 1960 (BARRETO et al., 2015; CAMPOLINA et al., 2013). As doenças crônicas são um importante problema de saúde, causam absenteísmo, incapacidade e ainda são responsáveis por $68 \%$ das mortes no mundo. Destas, $40 \%$ são consideradas prematuras, ocorrendo antes dos 70 anos (DUNCAN et al., 2012). Segundo a Organização Mundial de Saúde, até o ano de 2020, as condições crônicas serão responsáveis por $60 \%$ da carga global de doença nos países em desenvolvimento (MINAS GERAIS, 2006).

A Hipertensão Arterial Sistêmica (HAS) é uma DCNT de grande magnitude e um problema grave de saúde pública mundial, responsável por, pelo menos, $40 \%$ das mortes por acidente vascular cerebral e $25 \%$ das mortes por doença arterial coronariana. (OLIVEIRA, 2010). É uma síndrome que apresenta muitos fatores e causas e o seu aparecimento está intimamente ligado ao estilo de vida e relacionado a fatores constitucionais e ambientais (SOCIEDADE BRASILEIRA DE CARDIOLOGIA, 2013; REIS et al., 2012.). Por se tratar de uma doença "silenciosa" a HAS danifica os vasos sanguíneos renais, cardíacos e cerebrais e pode resultar em um aumento na incidência de insuficiência renal e cardíaca, coronariopatias e acidente vascular cerebral (SOUZA et al., 2010).

Brito \& Bortolotto (2011) afirmam que a HAS é a causa direta ou indireta de cerca de 7,5 milhões de mortes anualmente no mundo. Estimase que um em cada quatro adultos em todo o mundo pode ser classificado como hipertenso. Isso equivale a aproximadamente 1 bilhão de indivíduos hipertensos, sendo esperado um crescimento para 1,5 bilhão (cerca de $30 \%$ da população global) até 2025. Estima-se que a 
prevalência da HAS seja de $24,3 \%$ na população brasileira (BRASIL, 2013). Inquéritos populacionais em cidades brasileiras apontaram prevalência de HAS em indivíduos com 18-59 anos de idade, entre 20 a 30\%, chegando a atingir $50 \%$ na faixa etária de 60 a 69 anos e $75 \%$ em indivíduos com idade acima de 70 anos (MINAS GERAIS, 2013). Em Minas Gerais, a Secretaria de Estado da Saúde - SES/MG estima prevalência da HAS na ordem de $20 \%$ em sua população com idade igual ou superior a 20 anos (MINAS GERAIS, 2013)

Reis et al. (2012) ressaltam que, na população brasileira, a HAS apresenta um elevado custo socioeconômico, especialmente devido à sua importância na patogênese de doenças cerebrovasculares e doença arterial coronariana, entre outras. A HAS contribui com $60 \%$ das hospitalizações precoces no Brasil gerando custos de até R \$ 475 milhões por ano. Afirma-se que a HAS é responsável por $40 \%$ dos casos de aposentadoria precoce e de absenteísmo no trabalho no país (VEIGA et al., 2003).

De acordo com a Diretriz Brasileira de Hipertensão Arterial VII (2016), existem alguns fatores considerados de risco que, associados entre si e a outras condições, favorecem o desenvolvimento da HAS. São eles: fatores de risco não modificáveis: idade, gênero, etnia e antecedentes familiares (predisposição genética) e fatores de risco modificáveis (relacionados ao estilo de vida inadequado que por sua vez estão diretamente relacionados à maior prevalência dessa doença): obesidade, estresse, sedentarismo, alcoolismo, tabagismo, alimentação rica em sódio e gorduras (SOCIEDADE BRASILEIRA DE
CARDIOLOGIA, 2016; IBIAPINA et al., 2013; SOUSA, et al., 2012).

Desta forma, o presente estudo teve o objetivo de analisar as características sociodemográficas (sexo, faixa etária, escolaridade, raça/cor e situação conjugal) de indivíduos entrevistados, relacionando com a prevalência de HAS desses indivíduos na faixa etária de 18 a 46 anos na zona urbana de um município do Vale do Jequitinhonha, MG.

\section{MATERIAL E MÉTODOS}

Estudo transversal de base populacional, quantitativo, com a aplicação de questionários semiestruturados com questões fechadas e avaliação antropométrica de indivíduos na faixa etária de 18 a 46 anos, cadastrados na Estratégia de Saúde da Família na zona urbana do município Diamantina, MG, nos anos de 2014 e 2015. Este município possui uma população total de 45.880 habitantes (IBGE, 2010).

De acordo com os dados do Sistema de Informação de Atenção Básica (SIAB, 2013), 42.526 pessoas estavam cadastradas nas Estratégias de Saúde da Família - ESF do município em janeiro de 2013, sendo que estas integravam 11.322 famílias. $\mathrm{Da}$ população cadastrada na ESF do município, 27.970 pessoas $(65,8 \%)$ residiam na área urbana e destes 16.555 $(59,2 \%)$ eram adultos - com idade entre 15 e 49 anos.

$\mathrm{O}$ projeto e seus instrumentos foram aprovados pelo Comitê de Ética em Pesquisa da Universidade Federal dos Vales do Jequitinhonha e Mucuri, sob parecer $n^{\circ}$ 357.240; obtendo-se 
posteriormente a assinatura do termo de instituição copartícipe pela Secretaria Municipal de Saúde de Diamantina e do Termo de Consentimento Livre e Esclarecido (TCLE) pelos participantes. Foram consideradas as diretrizes e normas regulamentadoras envolvendo humanos, resolução CNS 466/12.

Para determinação do tamanho da amostra utilizada no estudo, utilizou-se a fórmula para estimação de proporção populacional considerando-se uma população finita, nível de confiança de $95 \%$ e erro de estimação tolerável de $4 \%$. Os indivíduos foram selecionados para participarem do trabalho levando-se em consideração a proporção de moradores por bairro em relação ao total de habitantes do município. Como no momento do cálculo do número de indivíduos a serem entrevistados por bairro aconteceram arredondamentos, o número final de sujeitos envolvidos neste trabalho passou a ser 571. Por existir nas unidades de saúde uma listagem nominal dos moradores do município que estão cadastrados nas ESF, o procedimento de sorteio adotado foi o de amostra sistemática estratificada (implicitamente) por área e microárea de abrangência da ESF, sexo e idade, sendo a população de estudo composta por moradores com idade entre 18 e 46 anos.

As entrevistas foram feitas na própria residência do participante. Foram realizadas pelo menos cinco tentativas de entrevistas em dias e horários diferentes e classificados como perda e substituídos os usuários que não foram localizados em uma das cinco visitas ou que se recusaram a participar do mesmo.
Após autorização da Secretaria Municipal de Saúde de Diamantina, MG, foi realizado nas oito ESF do Município, o levantamento dos nomes e endereços dos usuários na faixa etária entre 18 e 46 anos. Foram excluídas da amostra as gestantes e os portadores de incapacidades mentais ou cognitivas que inviabilizassem a resposta ao questionário.

Os participantes foram recrutados aleatoriamente, através de sorteio. Foi considerada a porcentagem de pessoas em cada bairro, dessa forma o sorteio foi feito por bairro e logo após cada indivíduo foi localizado na ESF para obtermos uma representação por bairro, já que as ESF abrangem mais de um bairro do município.

Utilizou-se como instrumento de coleta de dados, questionário padronizado do Ministério da Saúde - Secretaria de Vigilância em Saúde, Vigilância de Fatores de Risco e Proteção para Doenças Crônicas Não-Transmissíveis - Vigitel 2011 com adaptações. A escolha da faixa etária de 18 a 46 anos desse estudo foi devido à crescente prevalência da HAS em países em desenvolvimento e a escassez de estudos nessa faixa etária nas microrregiões do Brasil.

A prevalência de HAS foi calculada de acordo com o diagnóstico autorreferido pelos indivíduos entrevistados.

Após a coleta, os dados foram digitados, codificados e analisados. Para a entrada dos dados, foi utilizado o programa Excel 2010 e para as análises, $\mathrm{o}$ aplicativo livre $\mathrm{R}$ Core Team (2015). Para verificar se houve associação entre variáveis, foi adotado o nível de significância de 95\% ( $\mathrm{p}<0,05)$. A associação entre a prevalência 
de hipertensão arterial e as variáveis foi avaliada por meio do Teste de Qui-quadrado de Pearson ou pelo Teste Exato de Fisher com nível de significância de 0,05.

\section{RESULTADOS E DISCUSSÃO}

O presente estudo compreendeu as oito

ESFs da zona urbana de Diamantina, MG. A quantidade de usuários cadastrados nessas unidades na faixa etária entre 18 e 46 anos, bem como a quantidade e porcentagem dos sorteados, são apresentadas na Tabela 1 .

Tabela 1 - Quantidade de indivíduos cadastrados e sorteados por Estratégia de Saúde da Família (ESF) na zona urbana de Diamantina, MG, 2014-2015.

\begin{tabular}{|c|c|c|c|}
\hline ESF & Indivíduos cadastrados por ESF & \multicolumn{2}{|c|}{ Indivíduos sorteados por ESF } \\
\hline Sempre Viva & 1542 & 84 & 14,71 \\
\hline Bela Vida & 1939 & 106 & 18,56 \\
\hline Cazuza & 449 & 25 & 4,38 \\
\hline Encontro com a Saúde & 1133 & 62 & 10,86 \\
\hline Diamante e Vida & 1013 & 56 & 9,81 \\
\hline Renascer & 1518 & 83 & 14,53 \\
\hline Viver Melhor & 1249 & 68 & 11,91 \\
\hline Saúde e Vida & 1595 & 87 & 15,24 \\
\hline TOTAL & 10438 & 571 & 100,00 \\
\hline
\end{tabular}

Fonte: Próprio autor.

A prevalência de HAS foi calculada de acordo com o diagnóstico autorreferido pelos indivíduos entrevistados $(\mathrm{n}=55,9,6 \%)$. Esse diagnóstico foi avaliado quanto à resposta dos entrevistados à pergunta: "algum médico já lhe disse que você tem pressão alta?". Dessa forma, 9,6\% responderam sim, enquanto $90,4 \%$ deram resposta negativa. Vale ressaltar que fizeram parte dessa amostra adultos jovens. A análise dos dados permitiu-nos caracterizar a população de estudo de acordo com as características sociodemográficas e prevalência de HAS nesta. A Tabela 2 apresenta estes resultados.

A análise das características sociodemográficas apresentadas na Tabela 2, permite-nos observar que na população estudada a maioria era do sexo feminino, com $56,9 \%$ dos entrevistados, assim como o observado nos estudos de Nakashima et al. (2015) no Município de Tubarão, Santa Catarina, ao avaliarem o sexo dos participantes da pesquisa sobre fatores de risco para HAS na faixa etária entre 18 e 59 anos e nos estudos de Freitas et al. (2014) que também trabalharam com a investigação de fatores de risco para HAS. Ambos encontraram um predomínio do sexo feminino, com frequência de $(64,0 \%)$ e de $(61,5 \%)$ respectivamente, como no nosso estudo. De acordo com o Teste Quiquadrado de Pearson, podemos verificar que houve diferença significativa na prevalência de HAS em relação ao sexo dos entrevistados. A prevalência foi maior no sexo feminino $(12,0 \%)$. Dias et al. (2017), Galvão \& Soares (2016) e Malta et al. (2017) também constataram uma 
maior prevalência de hipertensão em mulheres de $29,4 \%, \quad 27,2 \%$ e $26,3 \%$ respectivamente. Almeida; Rodrigues (1997) e Silva et al. (2012) enfatizam a menopausa (caracterizada pela queda dos níveis hormonais de estrógenos e por uma série de alterações metabólicas, funcionais e estruturais), como um fator desencadeador da HAS em mulheres.

Tabela 2 - Características sociodemográficas e prevalência da Hipertensão Arterial Sistêmica (HAS) nos indivíduos entrevistados na Estratégia de Saúde da Família (ESF) da zona urbana de Diamantina, MG (n=571), 2014-2015.

\begin{tabular}{|c|c|c|c|c|c|}
\hline Variáveis & $\begin{array}{l}\text { Frequência } \\
\text { Absoluta }\end{array}$ & $\begin{array}{l}\text { Frequência } \\
(\%)\end{array}$ & Relativa & Prevalência (\%) & p-valor \\
\hline \multicolumn{6}{|l|}{ Sexo } \\
\hline Feminino & 325 & 56,9 & & 12,0 & \multirow{2}{*}{$\mathrm{p}<0,05 *$} \\
\hline Masculino & 246 & 43,1 & & 6,5 & \\
\hline \multicolumn{6}{|l|}{ Faixa Etária } \\
\hline 18 a 24 & 183 & 32,0 & & 2,7 & \multirow{4}{*}{$\mathrm{p}<0,001 *$} \\
\hline 25 a 34 & 220 & 38,6 & & 5,9 & \\
\hline 35 a 44 & 131 & 22,9 & & 13,7 & \\
\hline 45 a 54 & 37 & 6,5 & & 51,4 & \\
\hline \multicolumn{6}{|l|}{ Escolaridade } \\
\hline Fundamental incompleto & 44 & 7,7 & & 15,9 & \multirow{7}{*}{$\mathrm{p}<0,001 *$} \\
\hline Fundamental completo & 39 & 6,8 & & 23,1 & \\
\hline Médio incompleto & 138 & 24,2 & & 10,9 & \\
\hline Médio completo & 199 & 34,8 & & 8,0 & \\
\hline Superior incompleto & 56 & 9,8 & & 3,6 & \\
\hline Superior completo & 89 & 15,6 & & 5,6 & \\
\hline Mestrado ou doutorado & 6 & 1,1 & & 16,7 & \\
\hline \multicolumn{6}{|l|}{ Raça/cor } \\
\hline Branca & 120 & 21,0 & & 10,0 & \multirow{6}{*}{$\mathrm{p}<0,001 * *$} \\
\hline Negra & 115 & 20,1 & & 11,3 & \\
\hline Amarela & 3 & 0,5 & & 0,0 & \\
\hline Parda & 329 & 57,7 & & 9,1 & \\
\hline Indígena & 1 & 0,2 & & 0,0 & \\
\hline Não sabe & 3 & 0,5 & & $\ldots$. & \\
\hline \multicolumn{6}{|l|}{ Situação conjugal } \\
\hline Solteiro & 350 & 61,2 & & 4,3 & \multirow{6}{*}{$\mathrm{p}<0,001 * *$} \\
\hline Casado legalmente & 148 & 25,9 & & 18,2 & \\
\hline União estável & 54 & 9,5 & & 18,5 & \\
\hline Viúvo & 4 & 0,7 & & 25,0 & \\
\hline Separado/divorciado & 13 & 2,3 & & 15,4 & \\
\hline Não quis informar & 2 & 0,4 & & $\ldots .$. & \\
\hline
\end{tabular}

* Teste Qui-quadrado de Pearson. Significante se p-valor menor que 0,05. ** Teste Exato de Fisher. Significante se p-valor menor que 0,05 .

Fonte: Próprio autor.

A faixa etária mais prevalente neste estudo foi de 18 a 34 anos, representando $70,6 \%$ dos entrevistados. Dados semelhantes foram encontrados por Bezerra et al. (2013), em que a faixa etária predominante foi de 18 a 39 anos (46\%) e por Longo et al. (2011) ao delinearem a faixa etária mais frequente nos seus estudos $(20$ a 39 anos com frequência de 52,87\%). As análises estatísticas mostraram que quanto maior a idade, maior a prevalência da HAS na população de estudo, resultado que condiz com a Sociedade 
Brasileira de Cardiologia (2013) que afirma que existe relação direta e linear da PA com a idade.

A maior prevalência encontrada foi em pessoas com idade igual ou superior a 45 anos, sendo que estes dados se mostraram estatisticamente significativos. Nos estudos de Gonçalves et al. (2015) foi verificado elevação significativa da PA com o aumento da idade e Esperandio et al. (2013) verificou um aumento da prevalência de hipertensão com o aumento da idade. Além disso, nos estudos de Oliveira et al. (2015) houve associação entre hipertensão e idade superior a 45 anos, corroborando nossos resultados. Quanto à escolaridade, houve maior frequência de indivíduos com ensino médio completo (34,8\%), como o encontrado nos estudos de Ibiapina et al. (2013) em Teresina, Piauí, que encontrou a maior parte dos indivíduos em seu estudo (39,3\%) com ensino médio completo. Esta variável também apresentou diferença significativa em relação à prevalência de HAS, evidenciando neste estudo que quanto menor a escolaridade, maior a chance do indivíduo desenvolver HAS, assim como foi encontrado nos estudos de Piccini et al (2012), IBGE (2014), Aurélio et al. (2014) e Santos \& Mendez (2014) . É interessante observar que os indivíduos com escolaridade em nível de mestrado e doutorado apresentaram uma prevalência de HAS bastante superior àqueles com superior incompleto ou completo. É importante aprofundar os estudos a este respeito, aumentando-se o número de indivíduos avaliados, entretanto este fato talvez possa ser explicado em função de trabalhos que gerem mais estresse ao indivíduo, o que poderia levar ao quadro hipertensivo.
Segundo Duque et al. (2011), o sofrimento psíquico e o estresse ocupacional de pesquisadores são relevantes; eles têm que lidar com diversos elementos ansiogênicos e estressantes: carência de recursos para desenvolvimento das pesquisas, conviver em ambientes altamente competitivos, lidar com a pressão dos prazos e ainda conciliar atividades acadêmicas (atendimento à comunidade, docência e produção científica).

Quanto à raça/cor, houve predomínio da cor parda $(57,7 \%)$ entre os entrevistados, seguida da cor branca $(21,0 \%)$ e da cor negra $(20,1 \%)$. Rocha (2017) encontrou um predomínio de 53,4\% de indivíduos da raça/cor parda em seu estudo realizado no Município de Floriano, Piauí, corroborando nossos achados. Em relação à associação de prevalência de HAS em relação à raça/cor, o Teste Exato de Fisher mostrou diferença estatisticamente significativa, mostrando prevalências próximas para as raças negra $(11,3 \%)$, branca $(10,0 \%)$ e parda $(9,1 \%)$ de HAS. Mesmo próximas, a maior prevalência se deu em negros, como também citado na literatura por diversos autores. Dados semelhantes foram encontrados na Pesquisa Nacional de Saúde em 2013 apresentando prevalências de HAS 24\% para a raça negra, $22,1 \%$ para a branca e $20 \%$ para a parda (IBGE, 2014).

Os estudos de Bezerra et al. (2013) encontraram uma maior prevalência de HAS em negros $(45,3 \%)$, assim como Lessa et al. (2006) $(41,1 \%)$ em relação aos nossos resultados. Segundo Sousa et al., 2012, estudos realizados com populações afro-americanas têm sugerido maiores prevalência e gravidade da HAS em 
negros, podendo ser determinadas pela maior frequência de comprometimento em órgãos-alvo, maiores níveis de vasopressina plasmática e declínio na resposta renal neste grupo étnico.

$\mathrm{Na}$ literatura em estudos sobre hipertensão, a maior prevalência de hipertensão arterial em negros pode estar vinculada a fatores como a discriminação racial, que por sua vez está relacionado à baixa classe social, também pode ser justificado pela predisposição genética e até ao menos acesso aos serviços de saúde (Lessa, 2011).

Quanto à situação conjugal, houve maior frequência de solteiros $(61,2 \%)$, enquanto $35,4 \%$ eram casados legalmente ou possuíam união estável. Nos estudos de Souza et al. (2010), dentre os indivíduos entrevistados na faixa etária entre 40 e 90 anos, 65\% eram casados, $15 \%$ solteiros e $20 \%$ viúvos. Percebe-se que neste último, bem como em outros estudos, como o de Lima et al. (2014) em indivíduos com faixa etária entre 35 e 75 anos, Mendonça et al. (2012), com entrevistados com idade entre 31 e 82 anos e Amer et al. (2011), com indivíduos na faixa etária entre 18 e 89 anos, foram encontradas menores frequências de indivíduos solteiros (10\%, 24\% e $9,2 \%$ respectivamente), diferente dos nossos achados. Esse fato pode ser explicado pela faixa etária muito jovem da população de nosso estudo. A associação de prevalência de HAS em relação à situação conjugal se mostrou estatisticamente significativa pelo Teste Exato de Fisher, onde a maior prevalência desta doença se deu em indivíduos viúvos $(25,0 \%)$, seguido de indivíduos com união estável ou casados (18,5 e 18,2\%, respectivamente), a menor prevalência se deu em indivíduos solteiros (4,3\%). Resultados semelhantes foram observados por Sarmento et al. (2016) que encontraram maiores prevalências da HAS entre indivíduos viúvos $(56,3 \%)$ e casados $(27,5 \%)$ e menor prevalência entre os solteiros $(14,7 \%)$. Ferreira et al. (2009) constataram em seus estudos maior prevalência de HAS em indivíduos viúvos $(54,4 \%)$ e menor prevalência em indivíduos solteiros (10,6\%), corroborando nossos achados.

\section{CONCLUSÕES}

A prevalência de HAS na população estudada foi de 9,6\% (com diagnóstico médico autorreferido). Conclui-se também que nessa população a HAS atinge grupos socialmente vulneráveis, sendo bem mais prevalente no sexo feminino, com maior idade, menor escolaridade e da raça negra. É importante perceber que essa doença tem acometido também indivíduos de escolaridade mais avançada, provavelmente em virtude das suas ocupações. Ressalta-se a necessidade de conduzir trabalhos mais específicos voltados para essa população.

\section{REFERÊNCIAS}

\begin{abstract}
ALMEIDA, Fernando Antonio de; RODRIGUES, Cibele Isaac Saad. Revisäo/Atualizaçäo em Hipertensäo arterial: terapia de reposiçäo hormonal na menopausa: impacto sobre a pressäo arterial e outros fatores de risco cardiovasculares. J. bras. nefrol, v. 19, n. 4, p. 433-8, 1997.
\end{abstract}

AMER, Nadia Mohamed; MARCON, Sonia Silva; SANTANA, Rosangela Getirana. Índice de massa corporal e hipertensão arterial em indivíduos adultos no Centro-Oeste do Brasil. Arq Bras Cardiol, v. 96, n. 1, p. 47-53, 2011.

AURÉLIO, Marco; FONSECA, Vanessa; MENDONÇA, Danielle. Perfil epidemiológico dos pacientes com Hipertensão Arterial Sistêmica 
acompanhados por Programa Saúde da Família de São Sebastião-DF, Brasil. Rev. APS, v. 17, n. 3, 2014.BARRETO et al., 2015.

BEZERRA, Vanessa Moraes et al. Comunidades quilombolas de Vitória da Conquista, Bahia, Brasil: hipertensão arterial e fatores associados Quilombo communities in Vitória da Conquista, Bahia State, Brazil: hypertension and associated. Cad. Saúde Pública, v. 29, n. 9, p. 1889-1902, 2013.

BRASIL. Ministério da Saúde. Secretaria de Atenção à Saúde. Departamento de Atenção Básica. Estratégias para o cuidado da pessoa com doença crônica. Hipertensão Arterial Sistêmica. Cadernos de Atenção Básica, n 37. Brasília - DF, 2013.

BRASIL. Ministério da Saúde. Vigilância de fatores de Risco e Proteção para Doenças Crônicas por Inquérito Telefônico. Estimativas sobre frequência e distribuição sociodemográfica de fatores de risco e proteção para doenças crônicas nas capitais dos 26 estados brasileiros e no Distrito Federal em 2013. Vigitel, 2013.

BRITO, Thiago Midlej; BORTOLOTTO, Luiz Aparecido. Denervação renal no tratamento de hipertensão arterial resistente. Hipertensão, v. 18, n. 4, p. 145-8, 2011.

CAMPOLINA, Alessandro Gonçalves et al. A transição de saúde e as mudanças na expectativa de vida saudável da população idosa: possíveis impactos da prevenção de doenças crônicas. Cad Saúde Pública, v. 29, n. 6, p. 1217-29, 2013.

DA SILVA, Daniele Braz et al. Associação entre hipertensão arterial e diabetes em centro de saúde da família. Revista Brasileira em Promoção da Saúde, v. 24, n. 1, p. 16-23, 2012.

DE ARAÚJO MENDONÇA, Larissa Bento; LIMA, Francisca Elisângela Teixeira; DE OLIVEIRA, Sherida Karanini Paz. Acidente vascular encefálico como complicação da hipertensão arterial: quais são os fatores intervenientes? Escola Anna Nery Revista de Enfermagem, v. 16, n. 2, p. 340-346, 2012.

DE LUCENA, Eleazar Marinho Freitas et al. Análise do prognóstico de hipertensos cadastrados no HiperDia. Rev. bras. hipertens, v. 21, n. 3, p. 157162, 2014.

DIAS, Orlene Veloso et al. HIPERTENSÃO ARTERIAL SISTÊMICA AUTORREFERIDA: ESTUDO POPULACIONAL. Revista de APS, v. 20, n. $1,2017$.

DUNCAN, Bruce Bartholow et al. Doenças crônicas não transmissíveis no Brasil: prioridade para enfrentamento e investigação. Revista de saúde pública, v. 46, p. 126-134, 2012.

DUQUE, Juan Carlos; BRONDANI, Juliana Tabarelli; LUNA, Stélio Pacca Loureiro. Estresse e pós-graduação em Medicina Veterinária. Revista Brasileira de Pós-Graduação, v. 2, n. 3, 2011.

ESPERANDIO, Eliane Maria et al. Prevalência e fatores associados à hipertensão arterial em idosos de municípios da Amazônia Legal, MT. Rev Bras

Geriatr Gerontol, v. 16, n. 3, p. 481-93, 2013.

FERREIRA, Sandra Roberta Gouvea et al. Frequiência de hipertensão arterial e fatores associados: Brasil, 2006. Rev Saúde Pública, v. 43, n. 2, p. 98-106, 2009.

GALVÃO, Raphael Reis Silva; SOARES, Daniela Arruda. Prevalência de hipertensão arterial e fatores associados em adultos: uma revisão na literatura brasileira. Revista de APS, v. 19, n. 1, 2016.

GIBSON, Rosland S. Anthropometric assessment of body composition. New York: Oxford University Press. Principles of Nutritional Assessment, $2^{\mathrm{a}}$ ed. p. 187-207, 2005.

GONÇALVES, Talita Mota et al. Avaliação do nivel de percepção sobre hipertensão arterial de participantes atendidos em campanha de saúde: Petrolina, PE. Extramuros-Revista de Extensão da Univasf, v. 3, n. 1, 2015.

IBGE. Pesquisa Nacional de Saúde 2013. Percepção do estado de saúde, estilos de vida e doenças crônicas. Rio de Janeiro: Instituto Brasileiro e Geografia e Estatística, 2014.

IBGE. Um panorama da saúde no Brasil: acesso e utilização dos serviços, condições de saúde e fatores de risco e proteção à saúde 2008. Rio de Janeiro: Instituto Brasileiro e Geografia e Estatística, 2010.

IBIAPINA, Daniela Fortes Neves; SANTOS, Alessandra Nascimento; DE OLIVEIRA, Layenne Nathanna Rodrigues. Conhecimento dos pacientes com hipertensão arterial sobre a quantidade de sódio presente nos alimentos. Revista Interdisciplinar, v. 6, n. 4, p. 75-85, 2013.

LESSA I. Epidemiologia da hipertensão arterial sistêmica e insuficiência cardíaca no Brasil. Rev Bras Hipertens, v.8, n.4, p. 383-92, 2001.

LESSA, Ínes et al. Hipertensão arterial na população adulta de Salvador (BA)-Brasil. Arq Bras Cardiol, v. 87, n. 6, p. $747-56,2006$. 
LIMA, Edilson Rodrigues de; BARROS, Adriana Rolim Campos; OLIVEIRA, Camila Almeida Neves de. Percepção dos clientes hipertensos acerca das complicações da Hipertensão Arterial Sistêmica. Revista Interfaces: Saúde, Humanas e Tecnologia, v. 2, n. 5, 2014.

LONGO, Giana Zarbato et al. Prevalência e distribuição dos fatores de risco para doenças crônicas não transmissíveis entre adultos da cidade de Lages (SC), sul do Brasil, 2007. Rev. Bras.

Epidemiol, v. 14, p. 698-708, 2011.

MALTA, Deborah Carvalho et al. Prevalência e fatores associados com hipertensão arterial autorreferida em adultos brasileiros. Rev. Saúde Pública, v. 51, n. suppl 1, 2017.

MINAS GERAIS. Secretaria do Estado de Saúde. Atenção à Saúde do Adulto: Linha-Guia de Hipertensão Arterial Sistêmica, Diabetes Mellitus e Doença Renal Crônica. Belo Horizonte: SAS/ MG 2013.

MINAS GERAIS. Secretaria de estado de Saúde. Atenção à Saúde do idoso. Belo Horizonte: SAS/MG, 186 p, 2006.

NAKASHIMA, Leandro et al. Prevalência da Hipertensão Arterial Sistêmica em adultos do município de Tubarão (SC). Revista da AMRIGS, v. 59, n. 1, p. 4-9, 2015.

OLIVEIRA, Renata Aparecida Rodrigues de et al. Fatores associados à hipertensão arterial em professores da educação básica. Revista da Educação Física/UEM, v. 26, n. 1, 2015.

OLIVEIRA, Solange Glória de. Caracterização dos pacientes hipertensos e diabéticos atendidos em serviço de urgência e emergência no município de Dourados-MS. 2010. Tese de Doutorado. Escola Nacional de Saúde Pública Sérgio Arouca, 2010.

OMS. Cuidados Inovadores para Condições Crônicas Componentes. Organização Mundial da Saúde, Suíça 2002.

PICCINI, Roberto Xavier et al. Promoção, prevenção e cuidado da hipertensão arterial no Brasil. Rev Saúde Pública, v. 46, n. 3, p. 543-50, 2012.

REIS, Rodrigo R. et al. Tratamento de pacientes com hipertensão arterial sistêmica: influência da adesão e dos fatores de risco cardiovasculares. Medicina Acadêmica, v. 1, n. 2, 2012.

ROCHA, Maria Luciene; BORGES, José Wicto; MARTINS, Martha Fonseca Soares. Adesão ao tratamento da hipertensão arterial entre usuários da estratégia saúde da família em um municipio do piauí. Revista de APS, v. 20, n. 1, 2017.

SANTOS, Fernando Ribeiro; MENDEZ, Roberto Della Rosa. Estratificação de risco cardiovascular em hipertensos atendidos na atenção primária. Gestão e Saúde, v. 5, p. 2647-2658, 2014.

SARMENTO, Karen Costa et al. Fontes de obtenção de medicamentos para hipertensão e diabetes no Brasil: resultados de inquérito telefônico nas capitais brasileiras e no Distrito Federal, 2011. Cadernos de Saúde Pública, v. 32, 2016.

SOCIEDADE BRASILEIRA DE CARDIOLOGIA. Diretriz Brasileira de Hipertensão Arterial VII. Revista da Sociedade Brasileira de Cardiologia, Volume 107, No 3, Supl. 3, Setembro 2016.

\section{SOCIEDADE BRASILEIRA DE CARDIOLOGIA.} Diretrizes Brasileiras De Hipertensão VI. Revista Hipertensão, Volume 13, Número 1, 2013.

SOUSA, Hudson Wallença Oliveira et al. Portadores de hipertensão arterial: fatores de risco e prática farmacológica. Revista Tema, v.13, n.18-19, Campina Grande, dezembro, 2012.

SOUSA, Lara Emanueli Neiva de et al. Contribuições da produção científica da enfermagem sobre a subjetividade dos portadores de hipertensão arterial. Revista de Enfermagem da UFPI, v. 1, n. 1, 2012.

SOUZA, Geane Felix de; BRITO, Jackson Rabelo; FREITAS, Rivelilson Mendes. Aspectos epidemiológicos de pacientes hipertensos no município de Banabuiú. Cadernos da Escola de Saúde Pública, Ceará, v. 4, n. 1, p. 31-37, 2010.

VEIGA, Eugenia Velludo et al. Avaliação de técnicas da medida da pressão arterial pelos profissionais de saúde. Arq Bras Cardiol, v. 80, n. 1, p. 83-9, 2003.

\footnotetext{
Paola Aparecida Alves Ferreira

Mestre pelo programa de Pós Graduação em Saúde, Sociedade e Ambiente - PPGSaSA, na linha de pesquisa: Educação, Cultura e Saúde da Universidade Federal dos Vales do Jequitinhonha e Mucuri (UFVJM).
}

\section{Prof. Dr. Emerson Cotta Bodevan}

Doutor em Estatística pela Universidade Federal de Minas Gerais (2012). Atualmente é Professor Adjunto I da Universidade Federal dos Vales do Jequitinhonha e Mucuri. 
Prof. ${ }^{a}$ Dr. ${ }^{\text {a }}$ Leida Calegário de Oliveira

Doutora em Ciências Biológicas (Fisiologia e Farmacologia) pela Universidade Federal de Minas Gerais (2004). Atualmente é Professora Associado III da Universidade Federal dos Vales do Jequitinhonha e Mucuri e atuando como professora titular no PPGSaSA. 